\title{
ELECTRON BEAM SYSTEM OF THE TEVATRON ELECTRON LENS
}

\author{
N. Solyak, G. Kuznetsov, V. Shiltsev, FNAL, Batavia, IL 60510, USA \\ K.Bishofberger, UCLA, Los Angeles, CA 90095, USA
}

\section{Abstract}

The goal of the beam-beam compensation project at Fermalb is to improve performance of the Tevatron collider[1]. The first of the two Tevatron Electron Lens (TEL) for betatron tunes correction of individual antiproton bunches has been recently built, commissioned and installed in the Tevatron. The first series of studies [2] demonstrated a good agreement of the tuneshift of 980 $\mathrm{GeV}$ protons with theory.

We describe the electron beam system of the TEL, which includes vacuum system, electron gun, collector, ion and secondary electron cleaning electrodes, beam diagnostics (BPMs, current monitors, scrapers, wire scanner). The results of the electron beam studies are presented.

\section{GENERAL LAYOUT}

Fig.1 shows general layout of the TEL electron beam system. The total length of the TEL (flange-to-flange) is $3.65 \mathrm{~m}$, with the interaction length (zone inside SC solenoid, where electron and antiproton beams can overlapp) is about $2 \mathrm{~m}$.

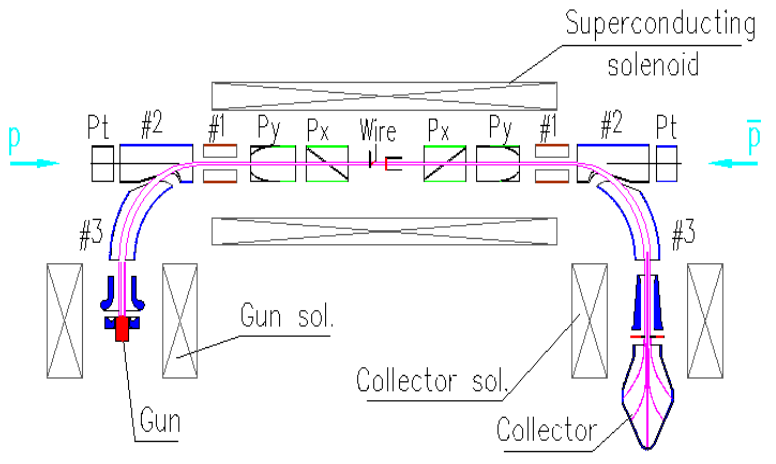

Figure 1. Electron beam system of the TEL.

The electron beam is born on the thermo-ionic cathode of the gun immersed in some $4 \mathrm{kG}$ magnetic field remains magnetized all the way to the to collector. $\Pi$ shaped magnetic system is formed by two conventional and one super-conducting solenoids. The electron beam in follows the magnetic force lines (magenta in Fig.1). Beam diameter in the main solenoid is defined by the cathode radius $a_{c}=5 \mathrm{~mm}$ and the ration of the magnetic field at the cathode $B_{c}$ and in the main solenoid $B$ :

$$
a=a_{c} \sqrt{B_{c} / B}
$$

Operational magnetic fields allow to have the electron beam size 3 times the antiproton $\mathrm{rms}$ size $\sigma=0.5 \mathrm{~mm}$, while at the same time the electron beam does not touch any apertures.
The typical TEL parameters are presented in Table1. Table 1: TEL operational parameters

\begin{tabular}{|lll|}
\hline electron beam energy, & $U_{e}, \mathrm{kV}$ & $6-12$ \\
maximum peak electron current & $J_{e}, \mathrm{~A}$ & $2-3.5$ \\
magnetic field in main solenoid & $B_{m}, \mathrm{kG}$ & 35 \\
\multicolumn{1}{c}{ in gun solenoid } & $B_{g}, \mathrm{kG}$ & 3.7 \\
e-beam radius in main solenoid & $a_{e}, \mathrm{~mm}$ & 1.75 \\
cathode radius & $a_{c}, \mathrm{~mm}$ & 5 \\
e-pulse width, FWHM & $\tau_{e}, \mathrm{~mm}$ & $\sim 800$ \\
repetition rate & $f_{\text {rep }}, \mathrm{kHz}$ & 47.7 \\
current stability, peak-to-peak & $\Delta J_{e} / J_{e}, \%$ & $<0.1$ \\
vacuum pressure & $e-8, \mathrm{Torr}$ & $2-8$ \\
\hline
\end{tabular}

The main SC solenoid has built-in dipole correctors for electron beam position and angle steering, see $[3,4]$. Additional 4 coils are built-in in the gun and collector solenoids and can be used as quadruple correctors of the beam ellipticity.

The beam diagnostics consist of two pairs (Px\&Py) of pick-ups (BPMs) $150 \mathrm{~mm}$ long and $\varnothing 70 \mathrm{~mm}$ each, located at the beginning and at the end of the interaction zone. Pick-ups made of diagonally cut SS cylinder for better linearity. BPMs can measure positions of electron, proton and antiproton beams. Gun and collector beam currents as well as beam losses on the scraper electrode at the collector entrance are measured by inductive coils (IC). Two wires can be remotely introduced into the center of the lens for the beam profile measurements in both $(\mathrm{X} \& \mathrm{Y})$ planes, see details in [5].

Fig. 2 shows the TEL electrical circuit. Vacuum beam pipe and pick-up electronics stay at the ground potential. Cathode and anode potentials are negative, typically $10 \mathrm{kV}$ to $-15 \mathrm{kV}$ and held by low-current DC power supply (V1) to compensate beam losses to the ground. Highcurrent power supply (V2) with potential up-to $+10 \mathrm{kV}$ drives cathode to anode beam current. To modulate the beam current three different types of $\mathrm{HV}$ modulators were used [6]. The modulating positive signal feeds the electron gun anode through a capacitor.

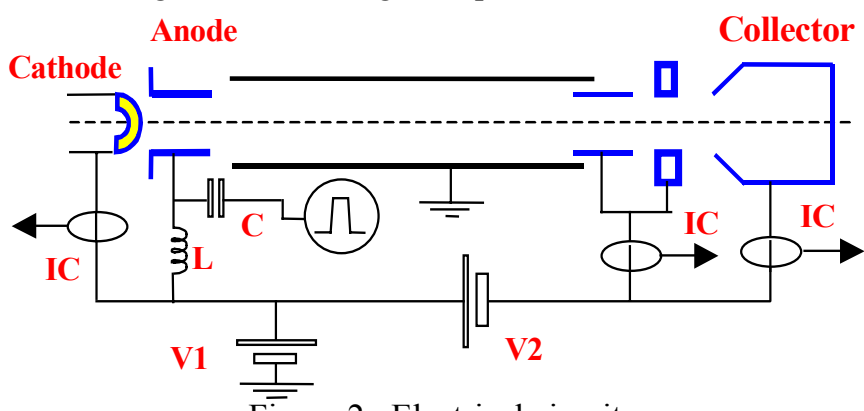

Figure 2. Electrical circuit.

Electron beam ionizes residual gas and if these ions and electrons are trapped and stored, their charge may change the lens performance. For cleaning the ions and electrons, 
several HV cleaning electrodes are installed. Two of them (\#1) are installed inside the main solenoid, each consist of tube $(\varnothing 70 \times 129 \mathrm{~mm})$ cut in half and these two halves are insulated by semi-conducting glass. Ions can escape if cleaning potential $( \pm \mathrm{U})$ strongly distorts the potential well due to the electron beam space charge [1]. Semiconducting glass avoid storing of the secondary electrons as well. Two elbow electrodes (\#3) in the bends work the same way. Electron beam size at that place is about 15$20 \mathrm{~mm}$, hence $\pm(5 \mathrm{kV}$ to $8 \mathrm{kV})$ of voltage thought to be enough for the cleaning. Cylindrical electrodes (\#2) provide ion cleaning in longitudinal direction by changing potential barrier. The cleaning procedure for the TEL is not studied well yet.

Beam size in the main solenoid depends on the magnetic field ratio (1), but the variation range is limited, as the beam can touch electrodes \#2 and \#3. The beam transmission is shown in Fig.3, loss-free zone is colored in dark-blue.

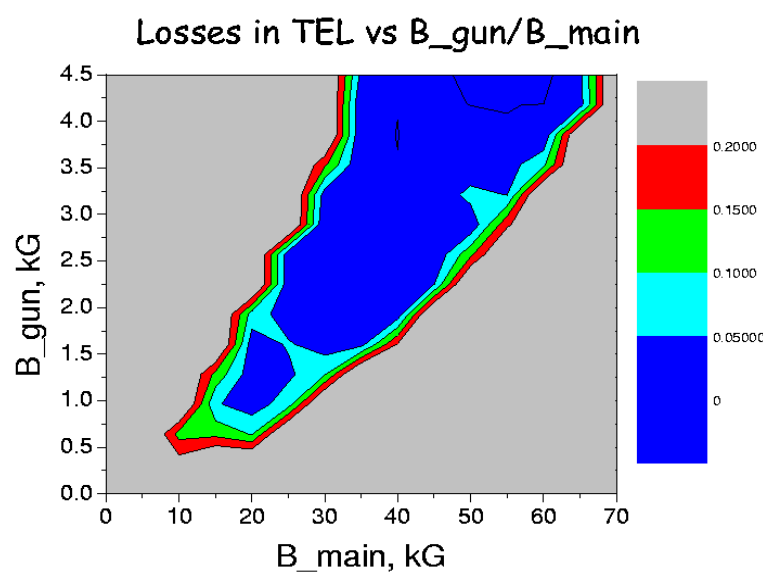

Figure 3. Plot of the electron beam transmission vs. magnetic fields $B_{\text {Gun }}, B_{\text {Main }}$.

\section{HIGH-PERVEANCE ELECTRON GUN.}

Electron gun -see Fig.4 - employs a $10 \mathrm{~mm}$ diameter convex cathode and can provide $10 \mathrm{~A}$ of pulsed current and about 3A DC. Measured perveance of the gun is

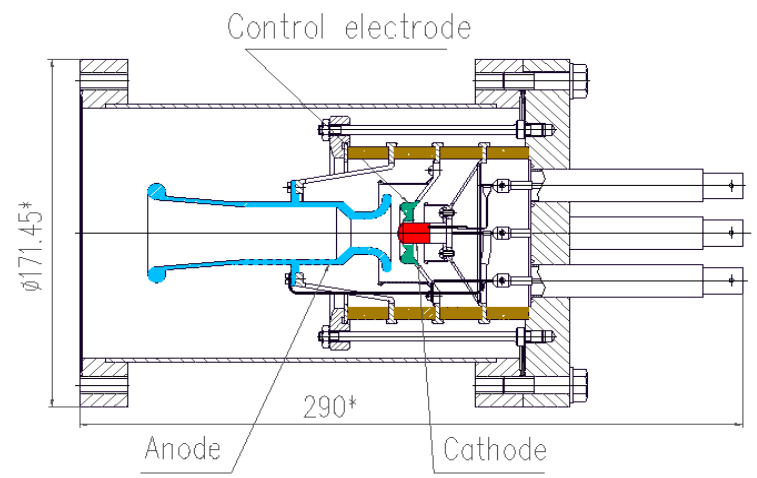

Figure 4. Electron gun assembly

$5.6 \mu \mathrm{A} / \mathrm{V}^{3 / 2}$, which is close to the design value [7]. Gun is assembled on 6.75" CF flange. All electrodes are insulated by 4 ceramic rings $(\mathrm{ID} \times \mathrm{OD}=79 \times 85 \mathrm{~mm}$ and $20 \mathrm{~mm}$ wide) and are constricted by stubs. The electrode capacitances are: $40 \mathrm{pF}$ anode to ground, $38 \mathrm{pF}$ anode to control electrode, $17 \mathrm{pF}$ anode to cathode. Vacuum HV feed-throughs provide $20 \mathrm{kV}$ DC to all electrodes. In presence of magnetic field the electrical strength may fall down to $15 \mathrm{kV}$ if the vacuum is worse than $5 * 10 \mathrm{e}-7$ Torr (due to Penning discharge). We plan to fix this problem in the improved gun design.

In pulsed regime the anode is driven by pulse modulator (see [6]). In the first tests a tetrode modulator with $800 \mathrm{~ns}$ and up to $7.5 \mathrm{kV}$ output pulse amplitude was used. Beam current vs. anode pulse voltage for $50 \mathrm{~Hz}$ and $50 \mathrm{kHz}$ (operational regime) repetition rates is shown in Fig.5.

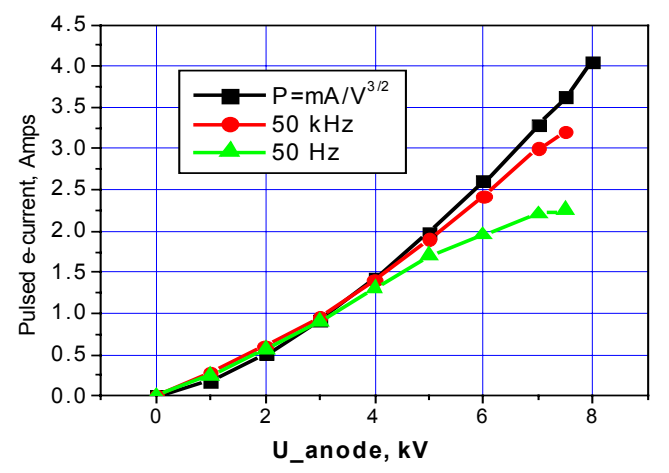

Figure 5. Beam current vs. anode voltage at $50 \mathrm{~Hz}$ and $50 \mathrm{kHz}$

The difference in behavior at low and high repetition rate can be explained by storing of ions in the case of $50 \mathrm{kHz}$. During $20 \mu \mathrm{s}$ between electron pulses the ions have not enough time to escape from the TEL and partly compensate the electron space charge. In this case, the beam current follows the Child's law. In absence of ions we have some restriction of electron current due to the beam pipe perveance.

Electron current density profile is almost flat [5] as required for the linear beam-beam compensation, but can be changed by applying negative potential to a special (control or "profiler") electrode near the cathode. In this case the beam profile becomes smoother (see [7]), while it reduces the total current and the beam size decrease, as shown in Fig.6. Beam with Gaussian profile is thought to be need for non-linear beam-beam compensation.

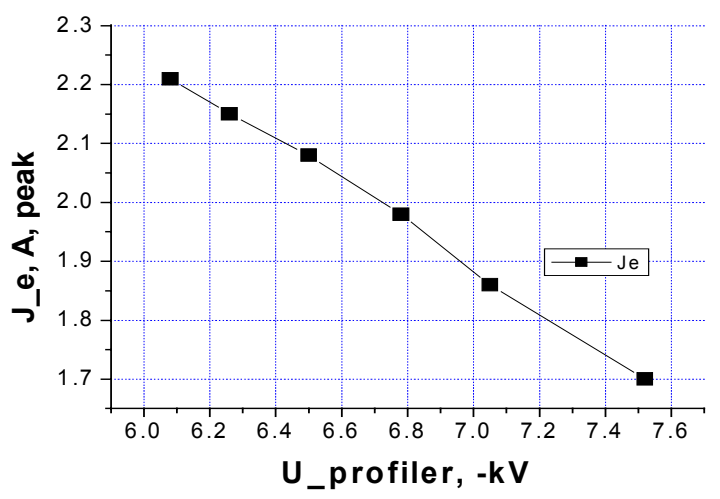

Figure 6. Electron current at $\mathrm{Uc}=-6 \mathrm{kV}$ vs. profiler voltage 
In the Tevatron Run IIa, the bunch spacing is $395 \mathrm{~ns}$. To be able to modulate electron current from bunch to bunch one needs a modulator with shorter pulse length.

Fig.7 shows 2A electron current (yellow) measured by the collector inductive coil and electron and proton bunches seen by BPM (green). Anode was driven by the tetrode modulator.

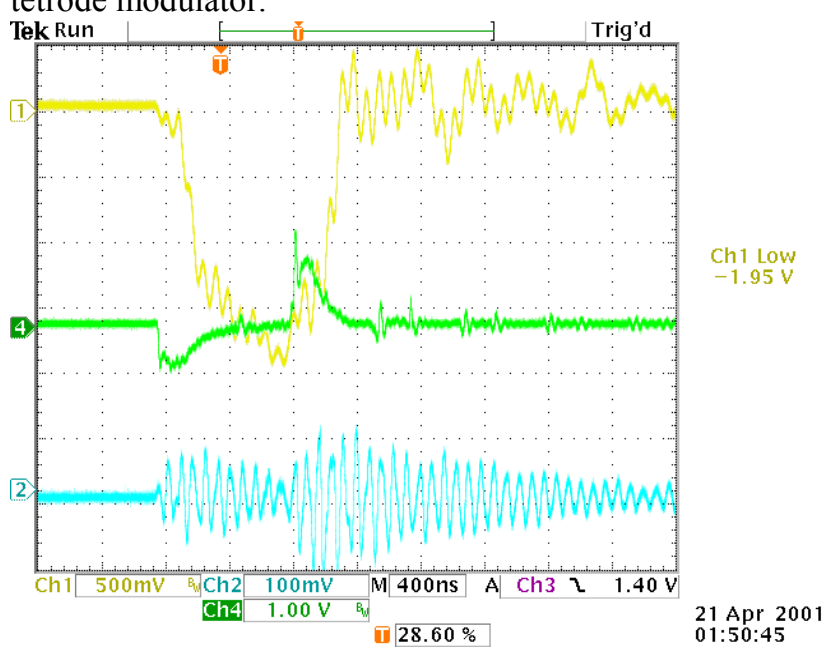

Figure 7. Oscilloscope trace of the electron current pulse.

\section{BEAM SIZE AND TRAJECTORIES}

Before installation of the beam diagnostics in the TEL, the beam trajectories as well as the beam size were measured with use of moveable $0.4 \mathrm{~mm}$ mesh screens. One of them did move along the axis of main solenoid, another - in the bend, as shown in Fig. 8.

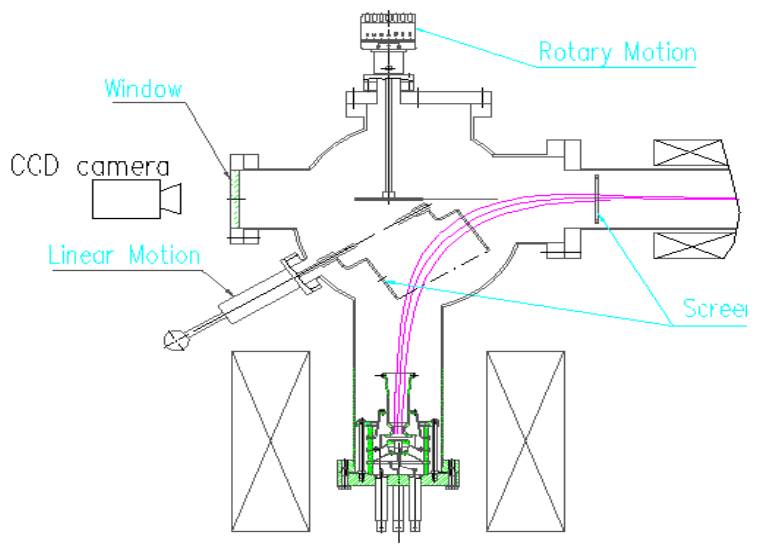

Figure 8. View screens for trajectories and beam size studying.

The electron beam image is read out through a glass window by CCD camera and stored in a PC. Beam image at the TEL center with the field ratio $B_{\text {main }} / B_{\text {gun }}$ $=35 \mathrm{kG} / 3.7 \mathrm{kG}$ is shown in Fig.9. Beam is moved on to the $4 \mathrm{~mm}$ wide edge of the screen. Beam has $\varnothing 3.5 \mathrm{~mm}$ with the hole inside, which can be explained by small perveance of the $\varnothing 113 \mathrm{~mm}$ beam pipe, which is only $\mu \mathrm{P}=3.4 \mu \mathrm{A} / \mathrm{V}^{3 / 2}$, i.e., much smaller than the gun perveance. With installed beam diagnostics $(\varnothing 70 \mathrm{~mm})$ and the beam post-acceleration, the gun and the pipe perveances can be matched.

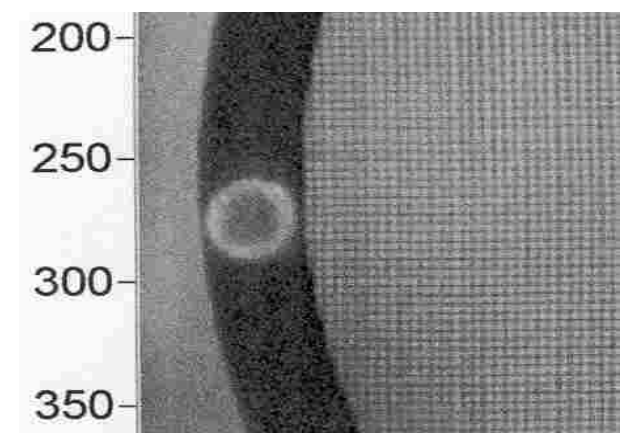

Figure 9. Electron beam image $(\mu \mathrm{P}=3.4)$.

\section{COLLECTOR}

Water-cooled collector, capable to dissipate $50 \mathrm{~kW}$ of the electron power was built in BINP (Protvino, Russia). It has high perveance of about $10 \mu \mathrm{A} / \mathrm{V}^{3 / 2}$ with absorbing efficiency $99.5 \%$ (see Fig.10). Collector is made of copper and insulated from the ground by a three-gap ceramics. Two additional insulated electrodes (entrance electrode and diaphragm) allow to measure beam losses during beam steering.

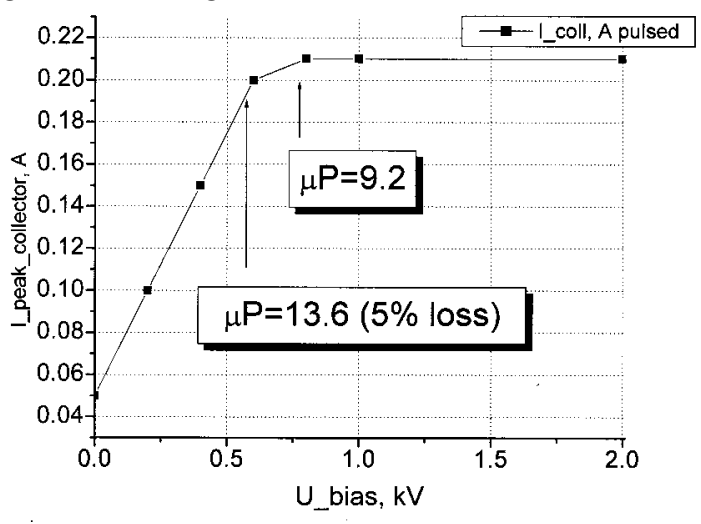

Figure 10. Collector beam current vs. voltage difference between cathode and collector

Diaphragm electrode was introduced to the collector design to suppress reflection of secondary electrons from collector, when negative potential of $1-2 \mathrm{kV}$ is applied.

\section{CONCLUSION}

Electron beam system of the TEL was designed, built and commissioned. Measured electron beam parameters are close to the designed ones. Planned gun upgrade will allow increase electron beam peak current.

We would like to thank A.Shemyakin and V.Dudnikov for valuable contribution to the TEL electron beam system design.

\section{REFERENCES}

[1] V.Shiltsev, et.al, Phys.Rev. ST-AB, 2,071001(1999)

[2] V. Shiltsev et al, this conference

[3] I. Bogdanov et al, FNAL-Conf-00/337(2000)

[4] K. Bishofberger. et. al, this conference.

[5] X. Zhang et. al, this conference

[6] D. Wildman et al, this conference.

[7] A. Shemyakin, et al, Proc.of EPAC 2000, p.1271 